\title{
OBSTRUCTIVE SLEEP APNEA SYNDROME AND THE QUALITY OF LIFE
}

\author{
ANDREEA CODRUTA COMAN ${ }^{1}$, CRISTINA BORZAN ${ }^{2}$, \\ CRISTIAN STEFAN VESA ${ }^{3}$, DOINA ADINA TODEA ${ }^{1}$
}

\author{
${ }^{1}$ Department of Pneumology, Iuliu Hatieganu University of Medicine and \\ Pharmacy, Cluj-Napoca, Romania \\ ${ }^{2}$ Department of Public Health, Iuliu Hatieganu University of Medicine and \\ Pharmacy, Cluj-Napoca, Romania \\ ${ }^{3}$ Department of Pharmacology, Toxicology and Clinical Pharmacology, Iuliu \\ Hatieganu University of Medicine and Pharmacy, Cluj-Napoca, Romania
}

\section{Abstract}

Background and aims. Obstructive sleep apnea syndrome (OSA) affects the quality of life (QOL) due to the effects on the patient's physical and mental function. QOL in sleep apnea may improve under continuous airway positive pressure (CPAP) therapy. The purpose of this study was to assess the OSA patients QOL before and after 3 months of CPAP therapy using Calgary Sleep Apnea Quality of Life Index (SAQLI).

Methods. We conducted a study in 79 sleep apnea subjects diagnosed using cardiorespiratory portable monitoring, under CPAP therapy, monitored in our Sleep Laboratory from January 2011 to December 2014. This is a cross-sectional study, achieved through quantitative research (SAQLI questionnaire application) about the perception of quality of life in patients with sleep apnea in the moment of diagnosis and 3 months after CPAP therapy.

Results. Of the 79 subjects, 59 (74.7\%) were men and 20 (26.3\%) women; mean age was 54.13 years (SD \pm 10.87 ), the mean apnea-hypopnea index (AHI) was $52.46 \pm 20.83$ events/h. In all 4 domains of SAQLI: daily functioning with mean pretreatment score $4.13 \pm 0.58$ versus mean post treatment score $5.43 \pm 0.52$; social interactions with mean pretreatment score $3.68 \pm 0.55$ versus post treatment mean score $5.36 \pm 0.57$; emotional functioning with mean pretreatment score $3.83 \pm 0.53$ versus mean post treatment mean $5.38 \pm 0.56$ and symptoms with mean pretreatment score $0.81 \pm 0.12$ versus mean post treatment score $1.15 \pm 0.14$, quality of life was improved after 3 months of therapy, with significantly statistical correlation $(p=0.00)$. Also, an improvement was seen in mean total score of SAQLI after therapy as compared to baseline $3.11 \pm 0.32$ versus $4.24 \pm 0.39(p<0.01)$.

Conclusion. The quality of life in sleep apnea was better after CPAP therapy than from baseline, according Calgary Sleep Apnea Quality of Life Index. The SAQLI is a useful toll to evaluate quality of life in sleep apnea, especially to highlight the benefits of CPAP therapy, even with short time monitoring.

Keywords: obstructive sleep apnea, quality of life, continuous positive airway pressure

Manuscript received: 07.10.2015

Received in revised form: 19.12.2015

Accepted: 07.01.2016

Address for correspondence: dede_coman@yahoo.com 


\section{Introduction}

Given the high prevalence of obstructive sleep apnea syndrome (OSA) and its effects on physical and mental function, the quality of life (QOL) in OSA has become a topic of interest in current literature. OSA is characterized by repetitive breathing stop episodes due to partial (hypopnea) or total (apnea) occlusion of the upper airways during sleep for at least 10 seconds; it is affecting $2 \%$ of middle age women and $4 \%$ of middle age men in developed countries [1], and may increase to $41 \%$ in obese patients [2]. Daytime sleepiness and loud snoring are the main diurnal versus nocturnal symptoms of OSA [1]. Recurrent oxyhemoglobin desaturation and arousal from sleep typical for OSA [3] entail health consequences in the cardiovascular area (pulmonary hypertension, resistant systemic hypertension, chronic heart failure, arrhythmia, myocardial infarction and stroke), increasing mortality [4], as well as in the neuropsychiatric area (depression, irritability, impaired cognition, difficulty of concentration or short-term memory loss, mood disorders), affecting familial, professional and social life and also increasing the risk for car and workplace accidents [5,6]. The CPAP (continuous positive airway pressure) the standard for OSA treatment, improves sleep quality, reduces daytime sleepiness, improves neurocognition and daily performance $[3,6,7]$.

Quality of life was defined by Schipper etal.: "Quality of life in clinical medicine represents the functional effect of an illness and its consequent therapy upon a patient, as perceived by the patient". There are also 4 main domains for measuring QOL: physical and occupational function, psychological function, social interaction and somatic sensation [8].

In OSA, the patient's affected quality of life may improve under CPAP therapy [7,9]. The rapid growth of interest in QOL has increased the number of available QOL instruments focused on OSA [5]. The Calgary Sleep Apnea Quality of Life Index (SAQLI) developed by Flemons and Reimer [10] seems to be the most complex tool to measure the outcomes and to cover all 4 domains for measuring QOL.

Numerous studies have been developed in various areas to assess QOL in OSA, such as in diagnosed patients and controls [11], diagnosed patients and bed partners [3], cross-sectional samples of patients [12,13], patients treated with CPAP [3] or with CPAP and uvulopalatoplasty [14]. Even if in the last years the interest in this topic has increased, the quality of life in OSA is not simple and not fully understood. Every new study in this area will increase the power of the "quality of life", providing arguments that the patient with OSA should be treated in a multimodal and holistic way focused beyond medical treatment in all physiological, emotional and social forms. Nevertheless, there are no studies regarding the quality of life in OSA patients in Romania. The purpose of this study was to assess the OSA patients QOL before and after CPAP therapy using SAQLI.

\section{Materials and methods}

This is a cross-sectional study, achieved through quantitative research (SAQLI questionnaire application) about the perception of quality of life in patients with sleep apnea at the moment of diagnosis and 3 months after CPAP therapy. Diagnosis of sleep apnea was made using cardiorespiratory portable monitoring.

Inclusion criteria: adult subjects, regardless of origin, religion and ethnicity, diagnosed with obstructive sleep apnea who accepted CPAP therapy at home and gave their informed consent to participate in the study.

Exclusion criteria: subject refusal to participate in the study, diagnosed mental and cognitive disorders, suspicion of mental retardation, acute psychosis.

\section{Subjects' enrollment}

The study was approved by the Ethics Committee of the Iuliu-Haţieganu University of Medicine and Pharmacy, Cluj-Napoca, Romania, prior to subject enrollment.

The 79 subjects included in this study were adult patients who referred from January 2011 to December 2014 to the Sleep Laboratory of Iuliu-Haţieganu University of Medicine and Pharmacy Cluj-Napoca, Romania. First, all the subjects were clinically evaluated, and those with symptoms were tested for OSA. Those diagnosed with OSA (nocturnal and diurnal symptoms and index of apnea hypopnea AHI $>5$ events/hour) and who agreed to CPAP therapy by signed informed consent were included in the study. After OSA diagnosis, each patient spent another night in the Sleep Laboratory for CPAP titration in order to establish the optimal pressure who eliminate snoring, hypopneas and apneas, and the pressure at which CPAP machines were to be set by home health care providers. A usage of CPAP $>4$ hours/night was considered acceptable. After the permission to use the original SAQLI version was obtained, the questionnaire was translated into the patients' native language by 2 authorized translators and was applied by the investigator to each patient before and after 3 months of CPAP therapy. Also for validation of the questionnaire a pre-testing was performed to a pilot group of 13 people, who were subsequently excluded from the survey. According to the study protocol, anthropometric, demographic, clinical data and medical history were collected from each subject.

\section{Measurement of the quality of life}

SAQLI is a specifically validated questionnaire for OSA which meets the criteria for internal consistency, validity and responsiveness. It was developed to highlight the symptoms and deterioration of functioning that are sensitive to changes. It has the great advantage to reflect the potential negative consequences of treatment, being primarily developed as an evaluative instrument, in order to measure change following treatment intervention. All these advantages of SAQLI are brought together in five domains of interest: A) daily functioning; B) social interactions; C) emotional functioning; D) symptoms; E) treatment-related symptoms. SAQLI was designed to be administered by an 
interviewer using a scale with 7 response options. The final score is obtained by the sum of the mean scores of each of the four domains, divided by four. Each domain score and the total SAQLI score have a possible score range from 1 to 7, which makes the score easier to interpret. Domains A-D are used to quantify pre-treatment QOL outcomes of OSA and domain $\mathrm{E}$ is used just for the QOL of CPAP treated patients $[10,15]$.

The Epworth Sleepiness Scale (ESS) is an eight-item questionnaire that measures excessive daytime sleepiness (EDS). This is defined as an ESS score $\geq 10$ (minimum $0=$ no fall asleep in any of the 8 situations and maximum $24=$ high probability to fall asleep in all the 8 situations) [16].

\section{Cardiorespiratory polygraphy (PG)}

Using a Philips Respironics Stardust II Sleep Recorder, cardiorespiratory polygraphy was performed for OSA diagnosis. All participants were recorded for at least $8 \mathrm{~h}$ and the variables were monitored using a data acquisition system for: nasal airflow with a nasal pressure transducer, thoracic and abdominal movements with two plethysmography belts, heart rate and oxygen saturation by pulse oximetry, body position and snoring. Polygraph records were scored manually. Diagnosis criteria for OSA were AHI $>5$ events/hour, daytime sleepiness or other symptoms [17]. The cut-off points for AHI were $5 \leq \mathrm{AHI}<15$ events/h, $15 \leq \mathrm{AHI}<30$ and $\mathrm{AHI} \geq 30$ to indicate mild, moderate, and severe levels of OSA. The oxygen desaturation index (ODI) is the number of times per hour of sleep that the blood oxygen level drops by 3 percent or more from baseline [18].

CPAP compliance was monitored in all participants in both subjective and objective ways. For the subjective collection of data, subjects were asked how many hours they spent with the CPAP machine every night. For objective data, a compliance card was downloaded, after data were recorded by a microprocessor in each central unit of the CPAP machine.

\section{Statistical Analysis}

Statistical analysis was performed using MedCalc Statistical Software version 15.8 (MedCalc Software bvba, Ostend, Belgium; https://www.medcalc.org; 2015). Quantitative variables were tested for normality of distribution using the Kolmogorov-Smirnov test. Quantitative variables were described using mean and standard deviation or median and 25-75 percentiles, whenever appropriate. Qualitative data was characterized by frequency and percent. Data variation between two measurements was performed using paired-t test, Wilcoxon test or ANOVA for repeated measures, whenever appropriate. The differences between groups were tested using Student $\mathrm{t}$ test, Mann-Whitney test, whenever appropriate. A $\mathrm{p}<0.05$ was considered statistically significant.

\section{Results}

All 79 subjects included completed the entire study. Proactive action from study coordinator via weekly telephones between basic and control regarding the CPAP night usage by each subject, was successful and led to a very good compliance. Of allpatients, 59 (74.7\%) were men and $20(26.3 \%)$ women with ratio male:female ratio7.47:2.53; the mean age was $54.13 \pm 10.87$ years, the mean neck circumference was $47.17 \pm 13.12 \mathrm{~cm}$; the mean abdominal circumference was $123 \pm 14.90 \mathrm{~cm}$; the mean body mass index (BMI) was $35.06 \pm 5.74 \mathrm{~kg} / \mathrm{m}^{2}$; the mean Epworth Sleepiness Scale (ESS) was 11.33 \pm 4.59 .

The data from the polygraphy recording and CPAP compliance from the card machine (after titration and after 3 months of CPAP therapy) are presented in Table I.

Table I. Characteristics from polygraphy and CPAP machine recording *.

\begin{tabular}{c|c}
\hline Characteristics & Values \\
\hline AHI (events/h) & $52.46 \pm 20.83$ \\
\hline Severe OSA (no./percent) & $68(86.1 \%)$ \\
\hline Moderate OSA (no./percent) & $10(12.7 \%)$ \\
\hline Mild OSA (no./percent) & $1(1.3 \%)$ \\
\hline ODI (events/h) & $50.90 \pm 21.23$ \\
\hline Medium SaO2 \% (median, percentile 25,75) & $91.00(88.00 ; 93.00)$ \\
\hline Minimum SaO2 \% (median, percentile 25,75) & $75.00(64.00 ; 83.00)$ \\
\hline $\begin{array}{c}\text { Residual AHI after CPAP titration; events/hour } \\
\text { (median, percentile 25,75) }\end{array}$ & $5.70(3.00 ; 7.40)$ \\
\hline CPAP pressure during titration (cm H2O) & $9.92 \pm 2.74$ \\
\hline CPAP compliance $>4$ hours of usage $\%$ (median, \\
percentile 25,75) & $57.50(55.00 ; 67.50)$ \\
\hline The median (hours) of CPAP utilization/night & $5.52(4.41 ; 7.04)$ \\
\hline
\end{tabular}

*Values given as mean $\pm \mathrm{SD}$. Data presented as median (50 percentile), also 25, 75 percentile because of non-normal distribution of the data. AHI: Apnea-hypopnea index; OSA: Obstructive sleep apnea; ODI: Oxygen desaturation index; $\mathrm{SaO} 2$ : Oxygen saturation; CPAP: Continuous airway positive. 
No statistical differences between males and females in QOL according to final score of SAQLI pre and post treatment was observed $(\mathrm{p}=0.44)$. Age did not influence the QOL evaluated by SAQLI ( $\mathrm{p}=0.38$ ). Also, there was no statistically significant difference between the risk factors for OSA like obesity and neck circumference $(p=0.5),(p=0.39)$ and the final score of SAQLI pre and post treatment. Subjects with symptoms of morning headaches had statistically significant $(\mathrm{p}=0.02)$ an improvement in QOL after 3 months of CPAP therapy evaluated by SAQLI (Figure 1). Other symptoms for OSA like apnea, $(\mathrm{p}=0.67)$, nighttime awakenings $(\mathrm{p}=0.98)$, choking during sleep $(p=0.70)$, restless sleep $(p=0.83)$, nocturia $(p=0.64)$ had no statistically significant correlation with SAQLI scores pre and post treatment. Regarding the comorbidities, those with chronic obstructive pulmonary disease (COPD) had an improvement on QOL at follow-up examination than on basics based on SAQLI $(\mathrm{p}<0.01)$ (Figure 2).

We found a statistically significant difference in AHI before CPAP therapy and after 1 night of titration $(p<0.01)$ and also between AHI from $1^{\text {st }}$ night of titration and AHI at 3 months of CPAP therapy ( $<<0.01)$. Also, subjects who used CPAP $\geq 4$ hours had statistically significant higher levels of SAQLI scores after 3 months of therapy $(p<0.01)$. OSA severity did not influence the QOL before and after 3 months of CPAP therapy $(\mathrm{p}=0.22)$.

Mean total score of SAQLI pretreatment was $3.11 \pm 0.32$ and mean total score of SAQLI post-treatment was $4.24 \pm 0.39$ with statistically significant difference $\mathrm{p}<0.01$, which means that QOL was improved at 3 months after CPAP therapy than from baseline.

In all 4 domains of SAQLI: A) daily functioning with mean pretreatment score $4.13 \pm 0.58$ versus mean post treatment score $5.43 \pm 0.52$; B) social interactions with mean pretreatment score $3.68 \pm 0.55$ versus post treatment mean score $5.36 \pm 0.57$; C) emotional functioning with mean pretreatment score $3.83 \pm 0.53$ versus mean post treatment mean $5.38 \pm 0.56$; D) symptoms with mean pretreatment score $0.81 \pm 0.12$ versus mean post treatment score $1.15 \pm 0.14$, all mean scores were improved after 3 months of therapy (Table II) with significantly statistical correlation $(\mathrm{p}=0.00)$. (Table III)

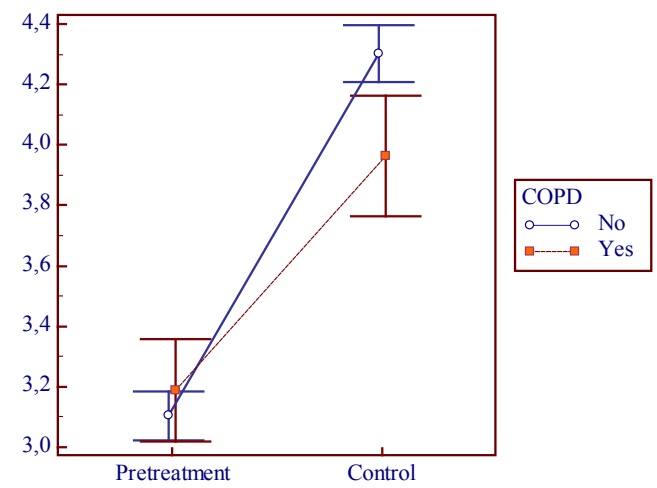

Figure 2. Pretreatment and control SAQLI values in COPD group. headaches group.

Morning headaches
$\square \quad 0$ No
$\square \quad$ Yes

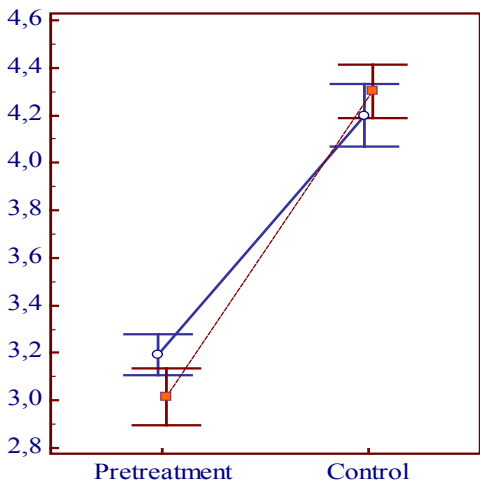

Figure 1. Pretreatment and control SAQLI values in morning

Table II. SAQLI score pre and post treatment in domains A) daily functioning; B) social interactions; C) emotional functioning; D) symptoms*.

\begin{tabular}{c|c|c|c|c|c}
\hline \multicolumn{2}{c|}{} & Mean & $\mathrm{N}$ & Std. Deviation & Std. Error Mean \\
\hline \multirow{2}{*}{ Pair 1 } & Score A p & 4.13 & 79 & .58 & .06 \\
\cline { 2 - 6 } & Score AP & 5.43 & 79 & .52 & .05 \\
\hline \multirow{2}{*}{ Pair 2 } & Score Bp & 3.68 & 79 & .55 & .06 \\
\cline { 2 - 6 } & Score BP & 5.36 & 79 & .57 & .06 \\
\hline \multirow{2}{*}{ Pair 3 } & Score Cp & 3.83 & 79 & .53 & .05 \\
\cline { 2 - 6 } & Score CP & 5.38 & 79 & .56 & .06 \\
\hline \multirow{2}{*}{ Pair 4 } & Score Dp & .81 & 79 & .12 & .01 \\
\cline { 2 - 6 } & Score DP & 1.15 & 79 & .14 & .01 \\
\hline
\end{tabular}

*Values given as mean $(\mathrm{SD}), \mathrm{n}=79$. Score $\mathrm{A} \mathrm{p}=$ pre treatment $\mathrm{SAQLI}$ score in domain $\mathrm{A}$; Score $\mathrm{A} \mathrm{P}=$ post treatment $\mathrm{SAQLI}$ score in domain A; Score B $\mathrm{p}=$ pre treatment SAQLI score in domain B; Score B P= post treatment SAQLI score in domain $\mathrm{B}$; Score $\mathrm{C} \mathrm{p}=$ pre treatment $\mathrm{SAQLI}$ score in domain $\mathrm{C}$; Score $\mathrm{C} \mathrm{P}=$ post treatment SAQLI score in domain $\mathrm{C}$; $\mathrm{Score} \mathrm{D} \mathrm{p}=\mathrm{pre}$ treatment $\mathrm{SAQLI}$ score in domain $\mathrm{D}$; Score $\mathrm{D} \mathrm{P}=$ post treatment $\mathrm{SAQLI}$ score in domain $\mathrm{D}$. 
Table III. Differences in SAQLI total scores pre and post treatment in all domains*.

\begin{tabular}{|c|c|c|c|c|c|c|c|c|c|}
\hline & & \multicolumn{5}{|c|}{ Paired Differences } & \multirow{3}{*}{$\mathrm{t}$} & \multirow{3}{*}{$\mathrm{df}$} & \multirow{3}{*}{$\begin{array}{c}\text { Sig. } \\
\text { (2-tailed) }\end{array}$} \\
\hline & & \multirow[t]{2}{*}{ Mean } & \multirow{2}{*}{$\begin{array}{c}\text { Std. } \\
\text { Deviation }\end{array}$} & \multirow{2}{*}{$\begin{array}{l}\text { Std. Error } \\
\text { Mean }\end{array}$} & \multicolumn{2}{|c|}{$\begin{array}{c}95 \% \text { Confidence Interval of the } \\
\text { Difference }\end{array}$} & & & \\
\hline & & & & & Lower & Upper & & & \\
\hline Pair 1 & Scor A p - Scor AP & -1.29 & .69 & .07 & -1.45 & -1.14 & -16.71 & 78 & .00 \\
\hline Pair 2 & Scor Bp - Scor BP & -1.67 & .86 & .09 & -1.86 & -1.47 & -17.21 & 78 & .00 \\
\hline Pair 3 & Scor Cp - Scor CP & -1.54 & .81 & .09 & -1.73 & -1.36 & -16.91 & 78 & .00 \\
\hline Pair 4 & Scor Dp - Scor DP & -.34 & .19 & .02 & -.38 & -.29 & -15.55 & 78 & .00 \\
\hline
\end{tabular}

*Correlation is significant at the $\mathrm{p}<0.05$ level (2-tailed)

The change in the total SAQLI score from pre to post intervention was 1.13 which is well above the minimal important difference and according to domains A,B,C and $\mathrm{D}$ the mean score difference was $1.29 \pm 0.69,1.67 \pm 0.83$, $1.54 \pm 1.54$ and $0.34 \pm 0.19$.

\section{Discussion}

The study was developed to test whether the quality of life in OSA, quantified using SAQLI, a specific questionnaire for sleep disorders, improved after CPAP therapy. To our knowledge, this research is the first to evaluate QOL in OSA after 3 months of CPAP therapy. We confirmed that after 3 months of CPAP therapy, QOL was improved in all domains of SAQLI: daily activities, emotional functioning, social interactions, symptoms and SAQLI visual scale, compared to baseline. The mean scores for all domains increased for the CPAP therapy groups compared to baseline values. Emotional functioning and social interactions had almost an equal impairment in OSA without CPAP and also, an almost equal improvement after CPAP therapy; daily activities were less affected in OSA at baseline and the improvement after therapy was significant; the symptoms domain was the worst affected; moreover, subjects chose themselves the 5 main affected symptoms. The obtained data are similar to those existing in the literature. For example, Dutt et al. [11] compared cases with controls and reported that daily functioning, social interaction and emotional functioning had an equal impairment and the symptoms domain was more affected. Parish and Lyng [3], in a recent study, compared the QOL of OSA patients and bed partners of patients with OSA after CPAP treatment, and found that QOL was impaired in both patients with OSA and their bed partners, and that CPAP treatment improved QOL measured by the SF-36 (ShortForm 36) and SAQLI. D'ambrosino et al. [19], using SF36 for measuring QOL, observed that all aspects of the quality of life, from physical and emotional health to social functioning, were impaired by OSA, and that CPAP therapy improved aspects related to vitality, social functioning and mental health. Moreover, in the Wisconsin Sleep Cohort
Study [20], even mild sleep breathing disorders were related to impairment of QOL according to SF-36.

Based on research data, subjects with COPD have a great improvement of QOL after CPAP therapy; this may be explained by the fact that CPAP therapy improves oxygen saturation levels during the night, which are lower in the case of an association between OSA and COPD; the positive daily consequences of this oxygen improvement are highest: a better QOL as evaluated by SAQLI. On the other hand, as seen in other studies, comorbid conditions may influence QOL; but in the study of Manocchia et al. [21], QOL was quantified using SF-36.

QOL in this study was affected in all domains, but we have to consider that most of the subjects had severe OSA, and also comorbidities, which may have been the reason of the great compliance with CPAP therapy. There are other studies in literature [3], in which many subjects were lost to follow-up or had a lower CPAP compliance. The category of subjects referred to the Sleep Laboratory with advanced symptoms, diagnosed with severe OSA and with a high level of CPAP compliance may have biased the study due to the fact that a group of patients who were more likely to comply with CPAP were included in the study, and this may not represent the entire OSA population. Indeed, efforts were made by the study coordinator through proactive weekly phone calls to maintain a proper attitude of the subjects regarding CPAP compliance; this could be a reason why subjects were compliant with the study.

In this study, OSA severity did not influence QOL evaluated by SAQLI. Similar results were reported in literature $[11,22]$. However, in a recent study by Lopes et al. [23], using SF-36 for OSA patients divided into nonphysically active and physically active and non-sleepy and sleepy, apneic patients with AHI $>30$ events/h who were sleepy and did not participate in physical activities had a decrease in all domains of SF-36 (physical functioning, role physical problems, bodily pain, general health perceptions, vitality, social functioning, emotional problems, general mental health).

ESS was used to assess only excessive daytime 
sleepiness for the subjects referred to our Sleep Laboratory, and the end point of our study was not to evaluate EDS by ESS after CPAP therapy, even if in other studies this evaluation was made [3] or the evaluation was made to compare cases with controls [11,23].

According to Lacasse et al. [24] OSA affects important domains of QOL, which remain unexplored in the sleep laboratory. The data of this study complement the conclusions of the previous authors, suggesting that the quality of life in OSA is impaired in all domains of patient perception about: somatic sensation, physical function, emotional state and social interaction [25], and grant, in addition to technical investigations in the Sleep Laboratory, a well deserved place to the concept of quality of life.

\section{Conclusions}

The quality of life in sleep apnea was improved after CPAP therapy than from baseline, according Calgary Sleep Apnea Quality of Life Index. The SAQLI is a useful toll to evaluate quality of life in sleep apnea, especially to highlights the benefits of CPAP therapy, even in terms short time monitoring. This topic, for the first time accessed in our country, provides quality of life researchers further evidence regarding the quality of life of adults with sleep apnea, from a geographical area that was not explored before. Conventional sleep recordings cannot provide details about the quality of life in OSA before and after CPAP therapy, that is why clinicians should focus on the quality of life measurements in their daily practice.

\section{Acknowledgements}

This paper was co-financed and published under the frame of the European Social Fund through the Sectorial Operational Programme Human Resources Development 2007-2013, project number POSDRU/107/1.5/S/ 82705.

\section{References}

1. McNicholas WT, Bonsignore MR. European Respiratory Monograph - Sleep Apnea. Eur Respir Mon. 2010;50:1-16.

2. Vgontzas AN, Tan TL, Bixler EO, Martin LF, Shubert D, Kales A. Sleep apnea and sleep disruption in obese patients. Arch Intern Med. 1994;154:1705-1711.

3. Parish JM, Lyng PJ. Quality of life in bed partners of patients with obstructive sleep apnea or hypopnea after treatment with continuous positive airway pressure. Chest. 2003;124(3):942-947. 4. Young T, Finn L, Peppard PE, Szklo-Coxe M, Austin D, Nieto FJ, et al. Sleep disordered breathing and mortality: eighteen-year follow-up of the Wisconsin sleep cohort. Sleep. 2008;31(8):10711078.

5. Moyer CA, Sonnad SS, Garetz SL, Helman JI, Chervin RD. Quality of life in obstructive sleep apnea: a systematic review of the literature. Sleep Med. 2001;2(6):477-491.

6. Epstein LJ, Kristo D, Strollo PJ Jr, Friedman N, Malhotra A, Patil SP, et al. Clinical guideline for the evaluation, management and long-term care of obstructive sleep apnea in adults. J Clin Sleep Med. 2009;5:263-276.
7. Flemons WW, Tsai W. Quality of life consequences of sleep-disordered breathing. J Allergy Clin Immunol. 1997;99(2):S750-S756.

8. Schipper H, Clinch JJ, Olweny CLM. Quality of life studies: definitions and conceptual issues. In: Spikler B (ed). Quality of life and pharmacoeconomics in clinical trials. 2nd ed. Philadelphia, PA: Lippincott-Raven; 1996: 11-23.

9. Flemons WW. Measuring health related quality of life in sleep apnea. Sleep. 2000;23 Suppl 4:S109-S114.

10. Flemons WW, Reimer MA. Development of a disease-specific health-related quality of life questionnaire for sleep apnea. Am J Respir Crit Care Med. 1998;158(2):494-503.

11. Dutt N, Janmeja AK, Mohapatra PR, Singh AK. Quality of life impairment in patients of obstructive sleep apnea and its relation with the severity of disease. Lung India. 2013;30(4):289-294.

12. Kingshott RN, Vennelle M, Hoy CJ, Engleman HM, Deary IJ, Douglas NJ. Predictors of improvements in daytime function outcomes with CPAP therapy. Am J Respir Crit Care Med. 2000;161(3 Pt 1):866-871.

13. Chervin RD, Aldrich MS. The Epworth Sleepiness Scale may not reflect objective measures of sleepiness or sleep apnea. Neurology. 1999;52(1):125-131.

14. Piccirillo JF, Gates GA, White DL, Schectman KB. Obstructive sleep apnea treatment outcomes pilot study. Otolaryngol Head Neck Surg. 1998;118(6):833-844.

15. Flemons WW, Reimer MA. Measurement properties of the calgary sleep apnea quality of life index. Am J Respir Crit Care Med. 2002;165(2):159-164.

16. Johns MW. A new method for measuring daytime sleepiness: the Epworth sleepiness scale. Sleep. 1991;14(6):540-545.

17. Sleep-related breathing disorders in adults: recommendations for syndrome definition and measurement techniques in clinical research. The Report of an American Academy of Sleep Medicine Task Force. Sleep. 1999;22:667-689.

18. American Academy of Sleep Medicine. International classification of sleep disorders. Diagnostic and coding manual. 2nd ed. Westchester: American Academy of Sleep Medicine; 2005.

19. D'Ambrosio C, Bowman T, Mohsenin V. Quality of life in patients with obstructive sleep apnea: effect of nasal continuous positive airway pressure--a prospective study. Chest. 1999;115:123-129.

20. Finn L, Young T, Palta M, Fryback DG. Sleep-disordered breathing and self-reported general health status in the Wisconsin Sleep Cohort Study. Sleep. 1998;21(7):701-706.

21. Manocchia M, Keller S, Ware JE. Sleep problems, healthrelated quality of life, work functioning and health care utilization among the chronically ill. Qual Life Res. 2001;10(4):331-345.

22. Yang EH, Hla KM, McHorney CA, Havighurst T, Badr MS, Weber S. Sleep apnea and quality of life. Sleep. 2000;23(4):535541.

23. Lopes C, Esteves AM, Bittencourt LR, Tufik S, Mello MT. Relationship between the quality of life and the severity of obstructive sleep apnea syndrome. Braz J Med Biol Res. 2008;41(10):908-913.

24. Lacasse Y, Godbout C, Sériès F. Health-related quality of life in obstructive sleep apnoea. Eur Respir J. 2002;19(3):499-503.

25. Shipper H, Clinch J, Powell V. Definition and conceptual issues. In: Spilker B (ed). Quality of life assessment in clinical trials. New York: Raven Press Ltd; 1990: 11-24. 